\title{
A framework for evaluating the effectiveness of protected areas: the case of Wolong Biosphere Reserve
}

\author{
Yihe Lü, Liding Chen, Bojie Fu*, Shiliang Liu \\ Key Lab of Systems Ecology, Research Center for Eco-Environmental Sciences, Chinese Academy of Sciences, \\ P.O. Box 2871, Beijing 100085, China
}

\begin{abstract}
To evaluate the effectiveness of protected areas, we developed a general framework to integrate necessary steps into an operational system. In this case study, we applied the framework to the Wolong Biosphere Reserve in Sichuan Province (southwestern China), the largest reserve of Giant Pandas (Ailuropoda melanoleuca). We collected data through questionnaire surveys and employed fuzzy modeling to analyze and integrate the data collected. The results revealed that Wolong Biosphere Reserve was moderately effective in nature conservation, especially in the protection of Giant Pandas. But the overall effectiveness of Wolong Biosphere Reserve was not as high as expected because its functions in environmental education, social development and economic development were only marginally effective. Both the overall effectiveness of Wolong Biosphere Reserve and the effectiveness of nature conservation need to be improved.

First, efforts should be directed towards improving nature conservation and more specifically on improving the protection of forest ecosystems. To achieve this objective, local farmers in Wolong Biosphere Reserve should become the main group improving nature conservation. In addition, more effective measures should be developed to raise the living standards and the socio-economic status of the local farmers in an environmentally compatible way.

Second, environmental education for local people and outsiders should be increased.

Furthermore, we need to strengthen basic research and build cooperation among the reserve administration bureau, researchers and other organizations at the local, national and international levels.

Finally, we need to improve the living conditions of the local population through education, family planning and healthcare. (C) 2002 Elsevier Science B.V. All rights reserved.
\end{abstract}

Keywords: Fuzzy modeling; Effectiveness; Protected areas; Wolong Biosphere Reserve

\section{Introduction}

The development of protected areas in China experienced four stages, starting with initiation, and followed by stagnation and devastation, restoration to the stage of rapid growth that began in the late 1970s (http://www.nre.com.cn/01/08.htm). The num-

\footnotetext{
* Corresponding author. Tel.: +86-10-62943840; fax: +86-10-62943840.

E-mail address: bfu@mail.rcees.ac.cn (B. Fu).
}

ber of protected areas increased from 34 in 1978 to 1276 in 2000, accounting for about 0.13 and $12.44 \%$ of the territory, respectively. The protected areas fall into three categories: natural ecosystems protection, wildlife protection, and natural relics protection (Xue and Jiang, 1994a). They are classified into national and local protected areas and supervised respectively by central and local administrative governmental agencies according to the 'Bylaw of protected areas of the People's Republic of China'. However, methods to evaluate the effectiveness of protected areas 
are not explained in the document. In practice, evaluating the effectiveness of protected areas is generally a neglected issue. Protected areas are not monitored routinely, and the general public do not know how effective they are. It is generally believed that natural resources, including the indigenous biota, are better protected from human disturbances in protected areas. However, the effectiveness of protected areas is limited by increasing human pressures (Liu et al., 1999). With the increasing number of protected areas, the quality of their management must be improved. Evaluating their effectiveness is a crucial step towards their sustainable management. To evaluate their effectiveness, we must first understand how protected areas function. To comprehend the functional status, we must develop an operational framework facilitating the mission of evaluation.

The major objectives of our study were to develop such an operational framework that could guide systematic and explicit evaluations of the effectiveness of protected areas in a simple manner, and to apply the framework to Wolong Biosphere Reserve, a typical and flagship Nature Reserve in China, currently criticized for its dysfunctions (Liu et al., 2001). Past research in protected areas concerned mainly the flora and fauna (Trakolis, 2001). Other research examined resource use (Samant et al., 2000) and conflicts between humans and protected areas (Maikhuri et al., 2000). The results of this research are necessary for the management of protected areas, but not sufficient for diagnosing problems or provision of a good decision support system for improving protected area management. Some researchers have described various methods for evaluating protected areas (Xue and Zheng, 1994; Hockings, 1998, 2000; Bruner et al., 2001; Liu et al., 2001). Some of the methods were largely descriptive and conceptual (Hockings, 1998) or simply got the results by scoring according to a predefined scale (Xue and Zheng, 1994; Hockings, 1998, 2000). It was clear what an evaluation should be from the detailed descriptions. But the methodology integrating the various aspects of an evaluation was out of reach of mere descriptions. The scoring system is based on the traditional crisp set theory. Crisp sets correspond to two-valued logic: yes or no, on or off, black or white, 0 or 1 (Bezdek, 1994). To complex systems, especially those with much imprecise and vague information, it may seem a little bit rigid. Simple statistics is a kind of quantitative method in assessing different aspects of protected areas (Bruner et al., 2001), however, it can hardly integrate the various aspects. Remote sensing and analysis is a powerful tool to provide quantitative assessment of land cover change (Liu et al., 2001), but not the causes of the change. Furthermore, what the management of protected areas faces is more than change in land cover. Therefore, by the method itself, we can seldom reach a full-scale evaluation of the effectiveness of the management of protected areas. Most of these problems are to be avoided through fuzzy modeling under the operational framework.

Following the framework development and implementation, we were able to distinguish the effective and ineffective aspects of protected area functions and determine the priorities for improving the management of protected areas. Our hope is that the framework and methodologies developed for our case study site can be applied to the evaluation of other protected areas in China and elsewhere. We also expect the results will provide important insights into the effectiveness of Wolong Biosphere Reserve and useful information for designing effective policies to improve the management of the reserve.

\section{Methods}

\subsection{The operational framework}

The evaluation process is composed of several important steps that form a continuous feedback loop (Fig. 1). Necessary data and the criteria for evaluation must first be collected and established according to the specific protected area of interest. Data collected can include quantitative or qualitative information.

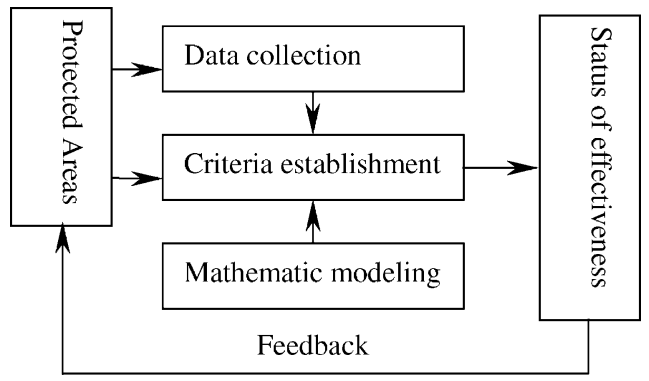

Fig. 1. The operational framework for evaluating the effectiveness of protected areas. 
Quantitative data can be obtained as remote sensing data, statistical data, land use data, and financial and staffing data. Qualitative data are usually collected by questionnaire surveys (Bruner et al., 2001; Kristensen et al., 2001; Trakolis, 2001) and household surveys (Shyamsundar and Kramer, 1997; Liu et al., 1999; Maikhuri et al., 2000). They provide information on human perceptions and other information beyond available quantitative data. The establishment of a criteria system is one of the key components of the evaluation process. Criteria systems may differ in some extent according to the specific characteristics of each protected area. With the data collected and the criteria system established, we can acquire information on the status of the effectiveness of protected area through mathematic modeling and analysis. The results are sent back to the management process for informing decision-making and policy development. Therefore, the framework proposed here can be used as an indispensable step in the adaptive management cycle of protected areas.

\subsection{The study area}

We chose Wolong Biosphere Reserve as our case study site because it is the largest nature reserve for Giant Pandas (Ailuropoda melanoleuca) and it is also typical of protected areas in China. The reserve was established in 1962 with an area of 20,000 ha and expanded to its current size of 200,000 ha in 1975 . It was established as a biosphere reserve of the reserve network of the Man and Biosphere Programme of UNESCO in 1980. Wolong Biosphere Reserve is located in Wenchuan County, Sichuan province, southwestern China $\left(102^{\circ} 52^{\prime}\right.$ to $103^{\circ} 24^{\prime} \mathrm{E}, 30^{\circ} 45^{\prime}$ to $\left.31^{\circ} 25^{\prime} \mathrm{N}\right)$. The reserve was established to protect alpine and sub-alpine ecosystems and endangered species such as Giant Panda, golden monkey (Rbinopitbecus spp.), and dove trees (Davidia involucrata). The reserve, with an elevation between 1200 and $6250 \mathrm{~m}$, is situated in the transition zone from Chengdu plain to
Qinghai-Tibet plateau. High mountains and deep valleys characterize this reserve. It maintains varied ecosystem types and a rich array of species.

The reserve is managed by the Wolong Administration Bureau. The Bureau reports to both China's Ministry of Forestry and Sichuan Province. There are two township governments under the Administration $\mathrm{Bu}-$ reau, Wolong Township and Gengda Township (Liu et al., 1999). In 2000, there were 1170 households in the reserve with a total population of 4515 . Local farmers account for about $86 \%$ of the total population. They depend mainly on traditional agriculture for livelihood.

\subsection{Data collection}

Human communities, especially those living in protected areas, often have important and long-standing relationships with these areas. Local peoples' perceptions contain useful information that could be incorporated into the decision-making process and lead to resolution of conflicts (Trakolis, 2001). Furthermore, community participation and equality need to be achieved urgently in decision-making process (IUCN, 1993). Otherwise, the costs and benefits of protected area management will not tend to be distributed proportionally at the site level, which is an important cause of various conflicts. Questionnaire surveys were used to collect data on the effectiveness of the reserve. Linguistic terms have been found intuitively easy to use in expressing the subjectiveness and imprecision of the decision maker's assessments (Zadeh, 1975; Deng and Yeh, 1998; Liang, 1999). Therefore, interviewees were asked to give their subjective assessments using pre-determined linguistic terms such as effective, marginally effective and so on (Table 1). The sampled population covered all the stakeholder groups in Wolong Biosphere Reserve including farmers, conservation workers, local research experts, governmental staff, administrators, teachers, workers in hydroelectric power and other industries, tourist bureau

Table 1

Triangular fuzzy numbers and the depicted linguistic terms used by decision makers

\begin{tabular}{llllll}
\hline Fuzzy number & $(9,9,7)$ & $(9,7,5)$ & $(7,5,3)$ & $(5,3,1)$ & $(3,1,1)$ \\
\hline Effectiveness $(V)$ & Highly effective & Rather effective & Effective & Marginally effective & Ineffective \\
Importance $(I)$ & Most important & More important & Important & Less important & Least important \\
\hline
\end{tabular}




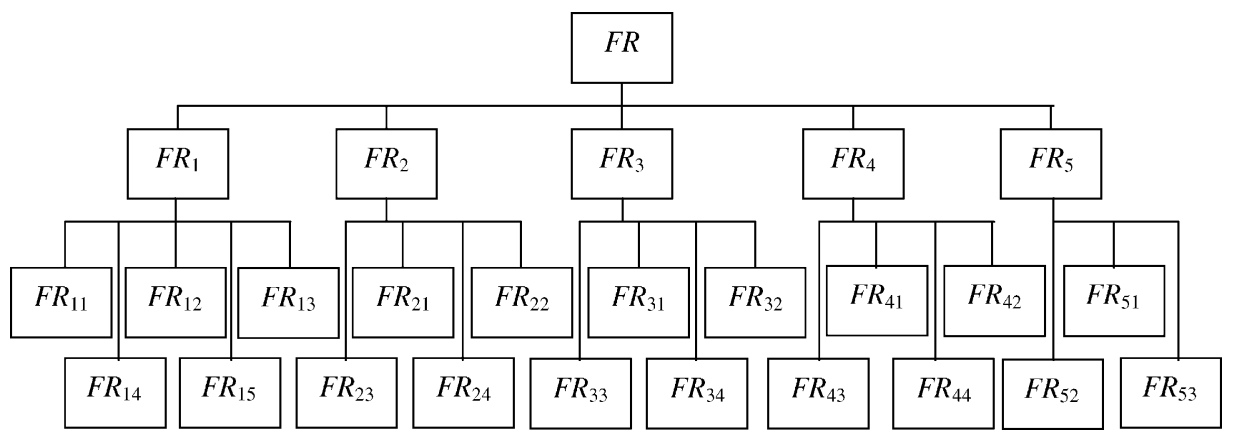

Fig. 2. The three-layer comprehensive evaluation criteria system. The strings in the textboxes represent the functions and the corresponding sub-functions of Wolong Biosphere Reserve. $\mathrm{FR}_{1}$ : nature conservation; $\mathrm{FR}_{11}$ : protection of Giant Pandas; FR 12 : forest ecosystem conservation; $\mathrm{FR}_{13}$ : landscape and natural resources conservation; $\mathrm{FR}_{14}$ : biodiversity conservation; $\mathrm{FR}_{15}$ : the protection of the total environment; $\mathrm{FR}_{2}$ : environmental education; $\mathrm{FR}_{21}$ : the infrastructure; $\mathrm{FR}_{22}$ : the measures; $\mathrm{FR}_{23}$ : effects on local residents; $\mathrm{FR}_{24}$ : effects on the outside; $\mathrm{FR}_{3}$ : scientific research; $\mathrm{FR}_{31}$ : the basis; $\mathrm{FR}_{32}$ : vigor and results; $\mathrm{FR}_{33}$ : cooperation and academic impacts; $\mathrm{FR}_{34}$ : research potential; $\mathrm{FR}_{4}$ : social development; $\mathrm{FR}_{41}$ : education; $\mathrm{FR}_{42}$ : culture; $\mathrm{FR}_{43}$ : social stability; $\mathrm{FR}_{44}$ : quality of population; $\mathrm{FR}_{5}$ : economic development; FR 51 : local economy; $\mathrm{FR}_{52}$ : economic efficiency of the reserve; $\mathrm{FR}_{53}$ : economic effects on the outside.

officers, doctors. The sampled interviewees were all above 18 years of age from different households with at least 9 years of education. Consequently, they could have a good understanding of the questionnaire and gave their answers properly and independently. In each questionnaire sample collected, criteria of the second and the third layer of the system were ranked in importance according to the upper layer criteria (Fig. 2) by the interviewee. Each criterion of the third layer was assessed with linguistic values. The average sampling ratio of different local stakeholder groups was about $10.8 \%$. There were some variation in the ratio according to the importance of the decisions of different stakeholder groups and the numbers in each group. Finally, a total of 170 questionnaires were completed and collected to form the database for the evaluation. Within the total, there were 32 from farmers, 26 from conservation workers, 16 from local research experts, 30 from governmental staff, 15 from administrators, 22 from teachers, 17 from workers in hydroelectric power and other industries, 10 from tourist bureau officers, and 2 from doctors.

\subsection{Data analysis}

\subsubsection{The establishment of the criteria system for evaluation}

Biosphere Reserves are internationally recognized protected areas with multiple functions such as conservation, research, monitoring, training, and sustainable development (Xue and Jiang, 1994b). Evaluating the effectiveness of protected areas has to be comprehensive enough to achieve rational and scientific conclusions. In order to evaluate the effectiveness of Wolong Biosphere Reserve, a systematic criteria system that reflects every aspect of the functions of the reserve is required. In this study, a three-layer comprehensive evaluation criteria system was proposed (see Fig. 2). In all, there are 5 criteria in the second layer and 20 criteria in the third layer.

Each of the third layer criteria has its distinctive meaning. Under the function of nature conservation $\left(\mathrm{FR}_{1}\right)$, the scope of conservation or protection grows wider and wider from a flagship species $\left(\mathrm{FR}_{11}\right)$ to forest cover $\left(\mathrm{FR}_{12}\right)$, all the living organisms in the reserve $\left(\mathrm{FR}_{13}\right)$, landscapes and other natural resources $\left(\mathrm{FR}_{14}\right)$, and finally the total environment $\left(\mathrm{FR}_{15}\right)$. Two criteria under the function of environmental education $\left(\mathrm{FR}_{2}\right)$ needs to be explained. The infrastructure $\left(\mathrm{FR}_{21}\right)$ refers to all the resources that a nature reserve needs to have for environmental education. The measures $\left(\mathrm{FR}_{22}\right)$ refer to the methods or channels used for environmental education. The basis for scientific research $\left(\mathrm{FR}_{31}\right)$ refers to all the resources that a nature reserve can provide for research work. Vigor and results of scientific research $\left(\mathrm{FR}_{32}\right)$ represent the situation of the research in operation and the outcome. $\mathrm{FR}_{33}$ refers to research cooperation between the nature reserve and 
other organizations (both local and international) and the impacts in academic fields. FR $_{34}$ refers to the capability of the reserve to support sustainable research in various fields of science. Under the function of social development $\left(\mathrm{FR}_{4}\right)$, education $\left(\mathrm{FR}_{41}\right)$ refers to the status of school education and culture $\left(\mathrm{FR}_{42}\right)$ refers to the maintaining of the traditional social culture. FR 44 refers to the living conditions of local population. Under the function of economic development $\left(\mathrm{FR}_{5}\right)$, local economy $\left(\mathrm{FR}_{51}\right)$ refers mainly to the economic development of local communities. Economic efficiency of the reserve $\left(\mathrm{FR}_{52}\right)$ refers to the reserve's input-output or capability of self-sustenance. Economic effects on the outside $\left(\mathrm{FR}_{53}\right)$ refer to the contributions of the reserve to the development of other regions.

\subsubsection{The models of fuzzy comprehensive evaluation and pattern recognition}

Fuzzy logic is a theory of uncertainty introduced by Zadeh (1965). It abandons the Aristotelian logic of two values and defines fuzzy sets as those whose confinements are not precise but vague. A fuzzy set is defined by assigning to objects of question membership grades to represent concepts to the whole (Zimmermann and Zysno, 1991). Fuzzy logic is a mathematical way to represent vagueness in everyday life. It is important because of the fact that most modes of human reasoning, especially common sense reasoning, are approximate in nature. Fuzzy interpretations of data structures are a natural and plausible way to formulate and solve various problems (Shaout and Al-Shammari, 1998). Its great utility has been found in control engineering and system analysis, knowledge engineering and expert systems, and artificial intelligence. This theory has the characteristic that it is able to represent the concepts of vagueness that natural language uses for pointing out qualitative variables (Enea and Salemi, 2001). The qualitative data or linguistic terms used to represent the subjective and imprecise assessments of the decision problem can be better modeled by fuzzy numbers (Yeh et al., 2000). In the present study, the linguistic values and the importance scale were depicted as triangular fuzzy numbers as $\left(a_{1}, a_{2}, a_{3}\right)$. Here $a_{1} \leq a_{2} \leq a_{3}$. The $a_{2}$ (Membership grade $=1$ ) is the most possible value of a linguistic term. The $a_{1}$ and $a_{3}$ are the lower and upper bounds (Membership grade $=0$ ) used to reflect the fuzziness of the term (Yeh et al., 2000). The fuzzy numbers used in current study and the corresponding linguistic terms are given in Table 1.

The universe of disclosure FR is the set of overall functions of Wolong Biosphere Reserve (Fig. 2), FR = $\left(\mathrm{FR}_{1}, \mathrm{FR}_{2}, \cdots, \mathrm{FR}_{m}\right)$ and $\mathrm{FR}_{i}$ is further composed of $n$ sub-functions, $\mathrm{FR}_{i}=\left(\mathrm{FR}_{i 1}, \mathrm{FR}_{i 2}, \cdots, \mathrm{FR}_{i n}\right)$. $V=\left(V_{j}\right)_{1 \times p}$ is the comment vector. The fuzzy decision matrix for the evaluation of $\mathrm{FR}_{i}$ is $R=\left(r_{i j}\right)_{n \times p}$, where $0 \leq r_{i j} \leq 1$ which represents the membership grades of the functions $\mathrm{FR}_{i j}$ to the linguistic assessments $V_{j}$ and can be calculated through some suitable membership function. The result matrix $B$ can be calculated as:

$B=A \circ R$

where $A=\left(a_{j}\right)_{1 \times n}$ is the weighting vector and ' $\circ$ ' is the generalized fuzzy operator for integration. The operator can take several forms according to different rules (Wu, 1994; Feng and Xu, 1999). As all the factors contributing to the reserve effectiveness are valued in the present study, we use the combination of ' $\bullet$ ' (multiplication sign) and ' $\sum$ ' (sum) denoted as $M\left(\bullet, \sum\right)$ as the operator. Similarly, taking $B$ as the decision matrix, we can figure out the result matrix of fuzzy comprehensive evaluation of the overall function FR. The membership function to be used here is constructed as $\mu(x)=n(x) / \sum n(x)$ from the characteristics of the data source. The $n(x)$ represents the number of questionnaires that gave the answer $x$. The decision matrices calculated from the membership function are shown in Table 2.

The weighting vectors can be calculated by the equation:

$W=F \frac{\mu(x)}{\sum F_{j}}$

where $F=\left(F_{j}\right)_{1 \times m}$ and $F_{j}$ is the most possible value of the fuzzy number in Table 1 . The weighting vectors are shown in Table 3. The score of effectiveness can be given by:

$E=F B^{\prime}$

where $E$ is the score of effectiveness; $B^{\prime}$ is the inversion of the result matrix $B$.

Suppose $S$, a vector, is the standard pattern. $T$, also a vector, is the pattern to be recognized. The recognition can be processed by Hamming neighborhood grade 
Table 2

The decision matrices for fuzzy evaluation

\begin{tabular}{|c|c|c|c|c|c|}
\hline Index & $V_{1}$ & $V_{2}$ & $V_{3}$ & $V_{4}$ & $V_{5}$ \\
\hline \multicolumn{6}{|l|}{$\mathrm{FR}_{1}$} \\
\hline $\mathrm{FR}_{11}$ & 0.2485 & 0.497 & 0.2071 & 0.0237 & 0.0118 \\
\hline $\mathrm{FR}_{12}$ & 0.213 & 0.3491 & 0.1775 & 0.1834 & 0.0533 \\
\hline $\mathrm{FR}_{13}$ & 0.1437 & 0.3593 & 0.2934 & 0.1497 & 0.0419 \\
\hline $\mathrm{FR}_{14}$ & 0.1761 & 0.3711 & 0.2642 & 0.1509 & 0.0314 \\
\hline $\mathrm{FR}_{15}$ & 0.1265 & 0.4277 & 0.2169 & 0.1627 & 0.0361 \\
\hline \multicolumn{6}{|l|}{$\mathrm{FR}_{2}$} \\
\hline $\mathrm{FR}_{21}$ & 0.0195 & 0.0779 & 0.0974 & 0.3442 & 0.2143 \\
\hline $\mathrm{FR}_{22}$ & 0.0132 & 0.0592 & 0.1053 & 0.4474 & 0.1776 \\
\hline $\mathrm{FR}_{23}$ & 0.0265 & 0.0464 & 0.1921 & 0.4106 & 0.1258 \\
\hline $\mathrm{FR}_{24}$ & 0.0195 & 0.0779 & 0.0974 & 0.3442 & 0.2143 \\
\hline \multicolumn{6}{|l|}{$\mathrm{FR}_{3}$} \\
\hline $\mathrm{FR}_{31}$ & 0.0839 & 0.1958 & 0.2238 & 0.2867 & 0.0769 \\
\hline $\mathrm{FR}_{32}$ & 0.1103 & 0.3379 & 0.2621 & 0.2138 & 0.0207 \\
\hline $\mathrm{FR}_{33}$ & 0.1563 & 0.1875 & 0.2969 & 0.2734 & 0.0547 \\
\hline $\mathrm{FR}_{34}$ & 0.3786 & 0.2429 & 0.15 & 0.1571 & 0.0214 \\
\hline \multicolumn{6}{|l|}{$\mathrm{FR}_{4}$} \\
\hline $\mathrm{FR}_{41}$ & 0.012 & 0.0898 & 0.1138 & 0.2934 & 0.1856 \\
\hline $\mathrm{FR}_{42}$ & 0.006 & 0.1138 & 0.1677 & 0.4132 & 0.1078 \\
\hline $\mathrm{FR}_{43}$ & 0.0361 & 0.2349 & 0.2952 & 0.3012 & 0.0663 \\
\hline $\mathrm{FR}_{44}$ & 0 & 0.0736 & 0.1104 & 0.2883 & 0.2638 \\
\hline \multicolumn{6}{|l|}{$\mathrm{FR}_{5}$} \\
\hline $\mathrm{FR}_{51}$ & 0 & 0.0189 & 0.1195 & 0.3145 & 0.2579 \\
\hline $\mathrm{FR}_{52}$ & 0.0265 & 0.0331 & 0.106 & 0.3444 & 0.2185 \\
\hline $\mathrm{FR}_{53}$ & 0 & 0.053 & 0.1515 & 0.3788 & 0.2045 \\
\hline
\end{tabular}

$\mathrm{FR}_{1}$ : nature conservation; $\mathrm{FR}_{11}$ : protection of Giant Pandas; $\mathrm{FR}_{12}$ : forest ecosystem conservation; $\mathrm{FR}_{13}$ : landscape and natural resources conservation; $\mathrm{FR}_{14}$ : biodiversity conservation; $\mathrm{FR}_{15}$ : the protection of the total environment; $\mathrm{FR}_{2}$ : environmental education; $\mathrm{FR}_{21}$ : the infrastructure; $\mathrm{FR}_{22}$ : the measures; $\mathrm{FR}_{23}$ : effects on local residents; $\mathrm{FR}_{24}$ : effects on the outside; $\mathrm{FR}_{3}$ : scientific research; $\mathrm{FR}_{31}$ : the basis; $\mathrm{FR}_{32}$ : vigor and results; $\mathrm{FR}_{33}$ : cooperation and academic impacts; $\mathrm{FR}_{34}$ : research potential; $\mathrm{FR}_{4}$ : social development; $\mathrm{FR}_{41}$ : education; $\mathrm{FR}_{42}$ : culture; $\mathrm{FR}_{43}$ : social stability; $\mathrm{FR}_{44}$ : quality of population; $\mathrm{FR}_{5}$ : economic development; $\mathrm{FR}_{51}$ : local economy; $\mathrm{FR}_{52}$ : economic efficiency of the reserve; $\mathrm{FR}_{53}$ : economic effects on the outside. $V_{1}$ : highly effective; $V_{2}$ : rather effective; $V_{3}$ : effective; $V_{4}$ : marginally effective; $V_{5}$ : ineffective.

(Chen, 1994), which is represented as:

$\delta_{\mathrm{H}}(S, T)=1-\frac{1}{n} \sum\left|S\left(u_{j}\right)-T\left(u_{j}\right)\right|$

Here, $\delta_{\mathrm{H}}(S, T)$ is the neighborhood grade between fuzzy vectors $S$ and $T ; n$ is the number of elements in each vector; $S\left(u_{j}\right)$ and $T\left(u_{j}\right)$ are the corresponding elements in vectors $S$ and $T$. In the present study the standard vector $S$ can be constructed following the
Table 3

Weighting vectors for fuzzy evaluation of the sub-functions of $\mathrm{FR}_{i}$ and the overall function FR

Weighting Elements of weighting vector vector

\begin{tabular}{llllll}
\hline$A_{\mathrm{FR}_{1}}$ & 0.3154 & 0.2034 & 0.1760 & 0.1469 & 0.1583 \\
$A_{\mathrm{FR}_{2}}$ & 0.3417 & 0.3060 & 0.1652 & 0.1571 & \\
$A_{\mathrm{FR}_{3}}$ & 0.3425 & 0.2708 & 0.2390 & 0.1477 & \\
$A_{\mathrm{FR}_{4}}$ & 0.2963 & 0.2714 & 0.2158 & 0.2164 & \\
$A_{\mathrm{FR}}$ & 0.4488 & 0.3604 & 0.1908 & & \\
$A_{\mathrm{FR}}$ & 0.2731 & 0.1856 & 0.1647 & 0.1649 & 0.2090 \\
\hline
\end{tabular}

rule: $S\left(u_{j}\right)=\max \left(r_{i j}\right), 1 \leq j \leq 4 ; S\left(u_{j}\right)=\min \left(r_{i j}\right)$, $4<j . T$ is the row vector in decision matrix $R . S$ is the ideal status which accommodates the best evaluation results to the functions in a given range.

\section{Results}

\subsection{The effectiveness of the functions of Wolong Biosphere Reserve}

The effectiveness of the functions of Wolong Biosphere Reserve are shown in Fig. 3. Using the scale developed by the fuzzy numbers in Table 1, we know the effectiveness of the functions clearly. The function of nature conservation is between effective and rather effective. The function of scientific research is effective. But the functions of environmental education, social development and economic development are marginally effective. Therefore, the overall function, the holistic representation of the functions in different respects, falls between marginally effective and effective.

Of the five functions, nature conservation is the most effective followed by scientific research, social development, environmental education, and economic development. Therefore, it is urgent for Wolong Biosphere Reserve to develop the local rural economy to a higher stage and promote environmental education. Educational approaches can be used as a tool in achieving conservation purposes (Papageorgiou, 2001). Economic development can inspire local farmers to protect the natural environment. Scientific research provides better understanding of the impacts and can improve environmental education, development and conservation. The function of nature conservation 


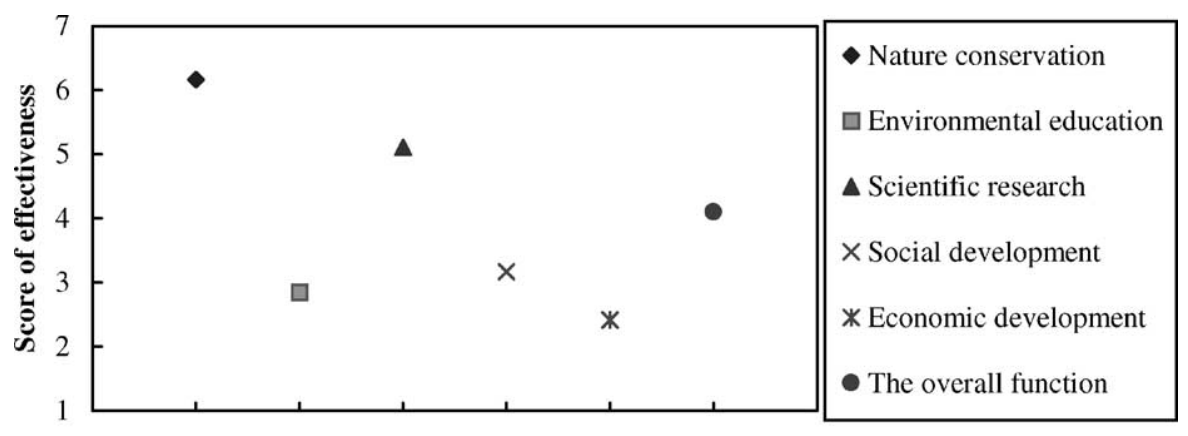

Function

Fig. 3. The effectiveness of the functions of Wolong Biosphere Reserve.

can be further improved only if the other four functions are promoted.

\subsection{The relative effectiveness and priorities of the sub-functions}

Under the function of nature conservation (Fig. 4), the first sub-function, the protection of Giant Pandas, is the most effective, followed by biodiversity conservation, landscape and natural resources conservation and the protection of the total environment. The least effective is forest ecosystems conservation. Therefore, to function better in nature conservation, the protection of forest ecosystems, a much criticized issue (Liu et al., 2001), must be strengthened first.

In environmental education (Fig. 5), the third sub-function (the effects on local people) is most effective, followed by the effects outside of the border, and environmental education measures used (the second sub-function). Infrastructure for environmental education, the first sub-function, is least effective. So the development of infrastructure for environmental education is a primary mission. At the same time the measures used for environmental education should be enriched and put into active use.

Under the function of scientific research (Fig. 5), Wolong Biosphere Reserve has a great potential of research in botany, zoology, conservation biology, ecology, and many other fields of science. The sub-functions including vigor and results of scientific research (the second sub-function) and research cooperation and academic impacts (the third sub-function) fall behind. The basis for scientific research, the first sub-function, is the poorest one. Accordingly, to improve scientific research, Wolong Biosphere Reserve needs to build up the basis for scientific research

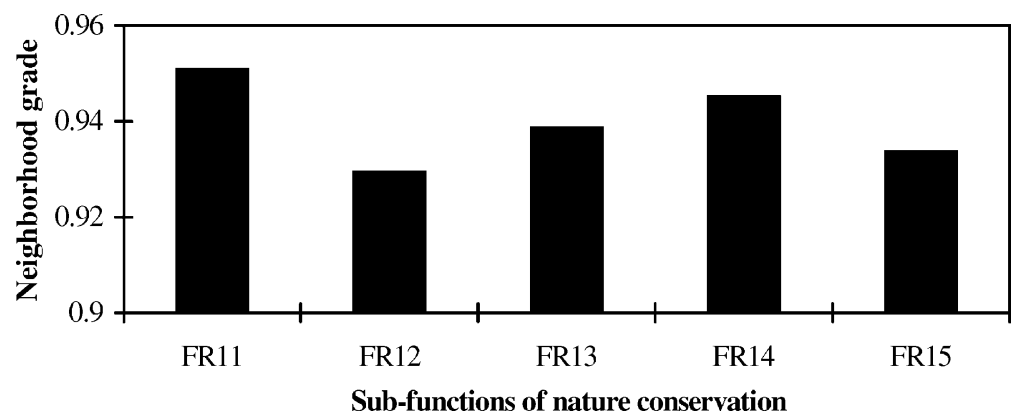

Fig. 4. The relative effectiveness of the sub-functions of nature conservation. FR $_{11}$ : protection of Giant Pandas; FR 12 : forest ecosystem conservation; $\mathrm{FR}_{13}$ : landscape and natural resources conservation; $\mathrm{FR}_{14}$ : biodiversity conservation; $\mathrm{FR}_{15}$ : the protection of the total environment. 


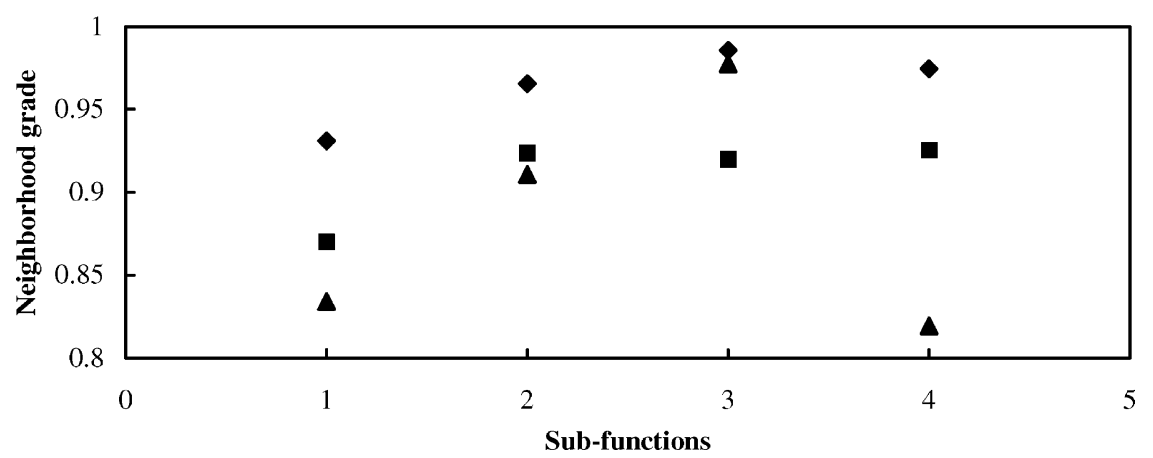

Fig. 5. The relative effectiveness of the sub-functions of environmental education $(\boldsymbol{\nabla})$, scientific research $(\boldsymbol{\square})$ and social development $(\boldsymbol{\Delta})$. $(\bullet)$ : 1, the infrastructure; 2, the measures; 3, effects on local residents; 4, effects on the outside. ( $\square$ ): 1, the basis; 2, vigor and results; 3, cooperation and academic impacts; 4, research potential. (A): 1, education; 2, culture; 3, social stability; 4, quality of population.

including all the materials needed in the process and to be more active in research cooperation, not only locally but also internationally with broader focus.

Under the function of social development (Fig. 5), Wolong Biosphere Reserve is most effective in keeping social stability (the third sub-function), followed by the development of local culture (the second sub-function), the development of education (the first sub-function), and the reserve is least effective in improving population quality (the fourth sub-function). Therefore, it is urgent for Wolong Biosphere Reserve to develop formal and informal education systems and to improve population health.

The relative status of the sub-functions of economic development are shown in Fig. 6. Wolong Biosphere Reserve is relatively effective as a source and sink of various goods and services, so it has positive effects beyond the border of the reserve $\left(\mathrm{FR}_{53}\right)$. The cost-effectiveness of the reserve $\left(\mathrm{FR}_{52}\right)$ falls behind.
And it is least effective in the development of local rural economy $\left(\mathrm{FR}_{51}\right)$. We find similar results as Wells (1992) found in his study of the distribution of the benefits and costs of protected areas at three spatial scales. Wolong Biosphere Reserve should pay more attention to the development of the local rural economy, including raising the living standards and creating more opportunities for local farmers to make a living in an environmentally beneficial way (such as the cultivation of medical plants or other plants with high market value, agro-forestry, and ecotourism). At the same time, more support in finance, policy, technology, and education should be given to the reserve to guarantee its sustainable development. In addition, anti-poverty programs are urgently in need within these programs.

The neighborhood grades of the sub-functions are shown in descending order in Fig. 7. The protection of Giant Pandas $\left(\mathrm{FR}_{11}\right)$ is the most effective of all the sub-functions, and local rural economic development

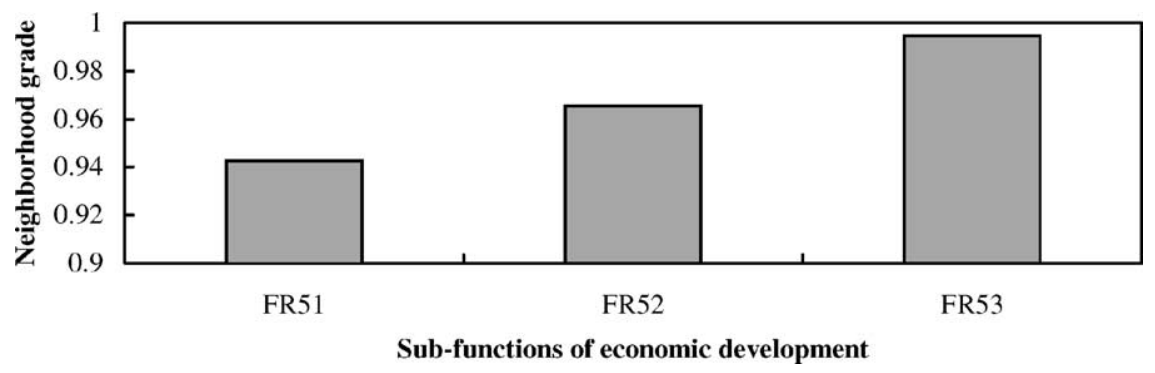

Fig. 6. The relative effectiveness of the sub-functions of economic development. $\mathrm{FR}_{51}$ : local economy; $\mathrm{FR}_{52}$ : economic efficiency of the reserve; $\mathrm{FR}_{53}$ : economic effects on the outside. 


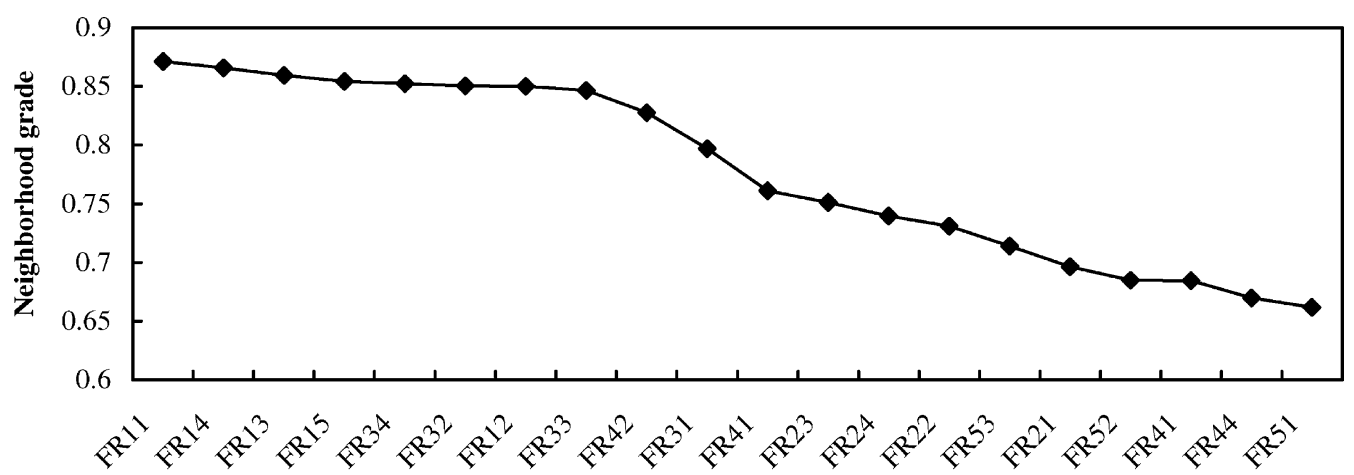

The sub-functions of the five reserve functions

Fig. 7. The overall ranking of the sub-functions against their neighborhood grades (the bigger the better). FR 11 : protection of Giant Pandas; $\mathrm{FR}_{12}$ : forest ecosystem conservation; $\mathrm{FR}_{13}$ : landscape and natural resources conservation; $\mathrm{FR}_{14}$ : biodiversity conservation; $\mathrm{FR}_{15}$ : the protection of the total environment; $\mathrm{FR}_{21}$ : infrastructure of environmental education; $\mathrm{FR}_{22}$ : measures of environmental education; $\mathrm{FR}_{23}$ : effects on local residents; $\mathrm{FR}_{24}$ : effects on the outside; $\mathrm{FR}_{31}$ : the basis for scientific research; $\mathrm{FR}_{32}$ : vigor and results of scientific research; $\mathrm{FR}_{33}$ : research cooperation and academic impacts; $\mathrm{FR}_{34}$ : scientific research potential; $\mathrm{FR}_{41}$ : development of education; $\mathrm{FR}_{42}$ : development of culture; $\mathrm{FR}_{43}$ : social stability; $\mathrm{FR}_{44}$ : quality of population; $\mathrm{FR}_{51}$ : development of local economy; $\mathrm{FR}_{52}$ : economic efficiency of the reserve; $\mathrm{FR}_{53}$ : economic effects on the outside.

$\left(\mathrm{FR}_{51}\right)$ is the least effective. The sub-functions with lower neighborhood grades need more attention and action in terms of management and policy.

\section{Discussion}

A criteria system was established based on the Biosphere Reserve Concept of UNESCO's Man and the Biosphere Programme and the researchers' understanding of the characteristics of Wolong Biosphere Reserve. The criteria system may differ between different protected areas, which is acceptable if it is scientifically sound. We used qualitative data in the fuzzy evaluation process in this study. The questionnaire data represents the perceptions and judgements of the interviewees. This type of data may result in evaluation errors due to differences between interviewees' knowledge and judgement scale. To minimize errors, we sampled all the stakeholder groups in Wolong Biosphere Reserve. There are differences in the decisions of various stakeholder groups and even the individuals from the groups. But no matter how different they are, the fuzzy modeling approach can integrate them without information loss. However, the evaluation results in this research may be more meaningful than reports provided by some 'experts' and governmental officers. Many of these other evaluations could be based on a short visit to the reserve for purpose of inspections and may not include the professional and long-term judgement of locals and field experts.

The evaluation results will be more dependable and convincing if adequate quantitative data are brought into the framework. To process the quantitative data, suitable membership functions and standards for classification need to be established. In this sense, our study so far can be regarded as a "rapid assessment". It is necessary, however, to conduct rapid assessments of protected areas in China due to limited human and financial resources. Our modeling approach provides a good basis for evaluating the effectiveness of protected areas and is currently ready for fine-tuning. Other protected areas can be evaluated following the steps of the approach used in this paper. If the scores of overall effectiveness of many protected areas are calculated, then we can rank the protected areas according to their scores. This can be helpful in urging the protected areas to improve their management.

We can see a general trend in the management of Wolong Biosphere Reserve. Nature conservation was stressed, while other functions such as development and education were de-emphasized. The protection of specific species has been given much importance, but the livelihood of local people and the development 
of local social-economy have been largely ignored. At the same time, protection and management at the ecosystem, landscape and even regional level mostly did not exist. These often led to policy failure and conflicts between protected area management and local peoples' livelihood needs. The trend does exist in the daily practice of the management of Wolong Biosphere Reserve. It determines the effectiveness of the functions and sub-functions of Wolong Biosphere Reserve and the overall effectiveness of the management of Wolong Biosphere Reserve in the long haul. This phenomenon is common in many other protected areas in China and deserves much attention.

\section{Conclusion}

Understanding the underlying mechanisms and inter-relationships of various functions of protected areas is critical to designing and implementing feasible policies for balancing conservation and development, which is a common challenge faced by protected areas or nature reserves around the world.

A protected area is a complex system. The functions are complementary and mutually reinforcing, or vice versa. They are subject to many factors as shown in this study. And the factors themselves can affect one another. The integrity of a protected area as a system and the issues of scale must be taken into consideration in the planning and management of protected areas. Although Wolong Biosphere Reserve is most effective in nature conservation, as our results have shown, the overall function of the reserve, affected by other four functions, is not so effective. Both the overall effectiveness of Wolong Biosphere Reserve and the effectiveness of nature conservation need to be improved.

Nature conservation is the most important function of Wolong Biosphere Reserve. Other functions must support this function but not undermine the importance of other functions. Therefore, more efforts in Wolong Biosphere Reserve should be made on nature conservation generally and forest ecosystem protection specifically. To achieve this purpose, we have to view local farmers in Wolong Biosphere Reserve as the main factor determining the state of nature conservation and provide more effective measures to raise their living standards and socio-economic status in an environmentally friendly way (such as the cultivation of medical plants or other plants with high market value, agro-forestry, and ecotourism). More support in finance, policy, technology and education is needed to build the capacity of the local people in conservation and development. Local participation is essential for the planning and management of the reserve.

Furthermore, we need to do enhance the environmental education of the local people and outsiders through the development of infrastructure and measures used in the process of environmental education. We should also improve scientific research through consolidating the basis for research and active participation in research cooperation, and improve the local population's quality of life through education, family planning and healthcare.

The fuzzy modeling used in this study is robust, compatible and flexible. It is highly adaptive to solving evaluation problems efficiently for protected areas. It is applicable not only to the evaluation of a single protected area but also to the evaluation of a series of protected areas simultaneously. Therefore, we recommend the use of fuzzy modeling in the evaluation process of protected area effectiveness. Following the operational framework developed in this research, we can both give an in-depth analysis of protected area effectiveness and make protected areas comparable in effectiveness.

\section{Acknowledgements}

This research was supported by the National Key Project for Basic Research in China (no. G2000046807). We are grateful to Wolong Nature Reserve administration Bureau and Mr. Qiu Jun for providing assistance in data collection. We thank all the interviewees for their cooperation in filling the questionnaires. Finally, we thank Dr. Cindy J. Lin for her constructive suggestions for revising the manuscript.

\section{References}

Bezdek, J., 1994. Fuzzy models-what are they, and why? In: Marks, R.J. (Ed.), Fuzzy Logic Technology and Applications: IEEE Technology Update Series. Technical Activities Board, Piscataway, NJ, pp. 3-7.

Bruner, A.G., Gullison, R.E., Rice, R.E., Fonseca, G.A.B., 2001. Effectiveness of parks in protecting tropical biodiversity. Science 291, 125-127. 
Chen, H., 1994. The Application of Fuzzy Mathematics in National Economy. Huazhong University of Science and Technology Press, Wuhan, China, pp. 72-77 (in Chinese).

Deng, H., Yeh, C.-H., 1998. Fuzzy ranking of discrete multicriteria alternatives. In: Proceedings of the IEEE Second International Conference on Intelligent Processing Systems. Gold Coast, Australia, pp. 344-348.

Enea, M., Salemi, G., 2001. Fuzzy approach to the environmental impact evaluation. Ecol. Model. 135, 131-147.

Feng, S., Xu, L.D., 1999. Decision support for fuzzy comprehensive evaluation of urban development. Fuzzy Sets Syst. 105, 1-12.

Hockings, M., 1998. Evaluating management of protected areas: integrating planning and evaluation. Environ. Manage. 22, 337345 .

Hockings, M., 2000. Evaluating effectiveness: a framework for assessing the management of protected areas. IUCN, Gland, Switzerland; Cambridge, UK. Available at http://Wcpa.iucn.org/ pubs/pdfs/Evaluating_Effect.pdf.

IUCN, 1993. Parks for Life. Report of the Fourth World Congress on National Parks and Protected Areas. IUCN, Gland, Switzerland, $252 \mathrm{pp}$.

Kristensen, S.P., Thenai, C., Kristensen, L., 2001. Farmers' involvement in landscape activities: an analysis of the relationship between farm location, farm characteristics and landscape changes in two study areas in Jutland, Denmark. J. Environ. Manage. 61, 301-318.

Liang, G.S., 1999. Fuzzy MCDM based on ideal and anti-ideal concepts. Eur. J. Operat. Res. 112, 682-691.

Liu, J., Ouyang, Z., Taylor, W.W., Group, R., Tan, Y., Zhang, H., 1999. A framework for evaluating the effects of human factors on wildlife habitat: the case of Giant Pandas. Conserv. Biol. 13, 1360-1370.

Liu, J., Linderman, M., Ouyang, Z., An, L., Yang, J., Zhang, H., 2001. Ecological degradation in protected areas: the case of Wolong nature reserve for Giant Pandas. Science 292, 98-101.

Maikhuri, R.K., Nautiyal, S., Rao, K.S., Chandrasekhar, K., Gavali, R., Saxena, K.G., 2000. Analysis and resolution of protected area-people conflicts in Nanda Devi Biosphere Reserve, India. Environ. Conserv. 27, 43-53.

Papageorgiou, K., 2001. A combined park management framework based on regulatory and behavioral strategies: use of visitors' knowledge to assess effectiveness. Environ. Manage. 28, 6173.

Samant, S.S., Dhar, U., Rawal, R.S., 2000. Assessment of fuel resource diversity and utilization patterns in Askot Wildlife Sanctuary in Kumaun Himalaya, India for conservation and management. Environ. Conserv. 27, 5-13.

Shaout, A., Al-Shammari, M., 1998. Fuzzy logic modeling for performance appraisal systems: a framework for empirical evaluation. Expert Sys. Appl. 14, 323-328.

Shyamsundar, P., Kramer, R., 1997. Biodiversity conservationat what cost? A study of households in the vicinity of Madagascar's Mantadia National Park. Ambio 26, 180184.

Trakolis, D., 2001. Local people's perceptions of planning and management issues in Prespes Lakes national park, Greece. J. Environ. Manage. 61, 227-241.

Wells, M., 1992. Biodiversity conservation, affluence and poverty: mismatched costs and benefits and efforts to remedy them. Ambio 21, 237-243.

Wu, B., 1994. Fuzzy Mathematics and its Application in Economic Analysis. Standards Press of China, Beijing, pp. 97-100 (in Chinese).

Xue, D., Jiang, M., 1994a. A study on categorizing standard of nature reserves in China. China Environ. Sci. 14, 246-251(in Chinese).

Xue, D., Jiang, M., 1994b. The Construction and Management of China's Nature Reserves. China Environmental Science Press, Beijing, pp. 25-38 (in Chinese).

Xue, D., Zheng, Y., 1994. A study on evaluation criteria for effective management of the nature reserves in China. Rural Eco-environ. 10, 6-9(in Chinese, with English abstract).

Yeh, C.-H., Deng, H., Chang, Y.-H., 2000. Fuzzy multicriteria analysis for performance evaluation of bus companies. Eur. J. Operat. Res. 126, 459-473.

Zadeh, L.A., 1965. Fuzzy sets. Inf. Control 8, 338-353.

Zadeh, L.A., 1975. The concept of a linguistic variable and its application to approximate reasoning (Parts I and II). Inf. Sci. 8, 199-249, 301-357.

Zimmermann, H.J., Zysno, P., 1991. Fuzzy Set Theory and its Application, 2nd ed. Kluwer Academic Publishers, Boston. 